\title{
Modelling gravity-driven flow over uneven surfaces
}

\author{
K. A. Ogden ${ }^{1}$, S. J. D. D’ Alessio ${ }^{1}$ \& J. P. Pascal ${ }^{2}$ \\ ${ }^{1}$ Department of Applied Mathematics, University of Waterloo, Canada \\ ${ }^{2}$ Department of Mathematics, Ryerson University, Canada
}

\begin{abstract}
This study concerns the gravity-driven two-dimensional laminar flow of a thin layer of fluid down a wavy inclined surface. Three mathematical models describing the unsteady two-dimensional flow evolution are presented and contrasted. The first is a shallow-water model, while the other two are integral-boundarylayer models representing non-hydrostatic approximations to the two-dimensional Navier-Stokes equations, which are valid for thin fluid layers. Various tests and simulations were conducted in order to assess the performance of the models. First, the instability threshold for the flat bottom case associated with each model was analytically determined and compared with the theoretical prediction based on the Navier-Stokes equations. Also for the flat bottom case, comparisons in neutral stability curves were made with existing experimental data. In addition, comparisons between two-dimensional numerical solutions of the full Navier-Stokes equations, obtained using the CFX software package, with simulations from the models were also investigated for a wavy bottom case. The wavy surface considered in this study corresponds to that of a sinusoidal profile. The emerging interfacial wave structure along with the combined effect of bottom topography and surface tension are discussed. Finally, critical Reynolds number predictions for cases including bottom topography are compared to existing experimental data.

Keywords: film flow, wavy incline, shallow-water and integral-boundary-layer models, numerical, experimental, analytical, CFX solver.
\end{abstract}

\section{Introduction}

There are many situations in which a model for flow down an inclined plane is applicable. Naturally occurring situations include mudslides and ice channels [1]. 
As well, such models are useful for engineering applications such as aqueducts, dam spillways, and coatings in manufacturing [2]. Analytic models for this type of flow are useful because they help predict key features of the flow, such as under what conditions it will become unstable and how the shape of the free surface will develop after this happens. These are important predictions to make because the large roll waves that are formed when the flow becomes unstable can overflow channel walls or damage measurement equipment in engineering settings; in natural settings, these roll waves result in more destructive surges of flow due to the increased mass flow rate and momentum [2]. Therefore, models that give accurate predictions of these features of the flow are important, and could be used to design channels that can handle the waves that are likely to occur, or that can reduce the likelihood of roll waves forming.

Many previous studies focus on the case without bottom topography. Detailed analytical investigations include those by Ruyer-Quil and Manneville [3], who developed models based on weighted residual methods. They consider even-bottom surfaces, and develop first- and second-order models applicable to two and three dimensional cases. The problem has also been extended by Balmforth and Mandre [1], and D'Alessio et al. [2] to include bottom topography. Balmforth and Mandre use the shallow-water model, focussing on the turbulent version, while D'Alessio et al. apply a weighted residual model to the uneven bottom case.

In this study, three models that describe fluid flow over an uneven, inclined plane are compared. The problem setup and coordinate system are shown in Figure 1. These models are the shallow-water model (SWM), the integral-boundarylayer (IBL) model, and the weighted residual model (WRM). Model predictions for the critical Reynolds number for the onset of instability are compared with the theoretical value obtained from the Orr-Sommerfeld equation for the flat bottom case. Neutral stability curves resulting from the models are also compared with experimental data for the flat bottom case. As well, fully developed flows for various sets of parameters are compared with direct numerical simulations of the full

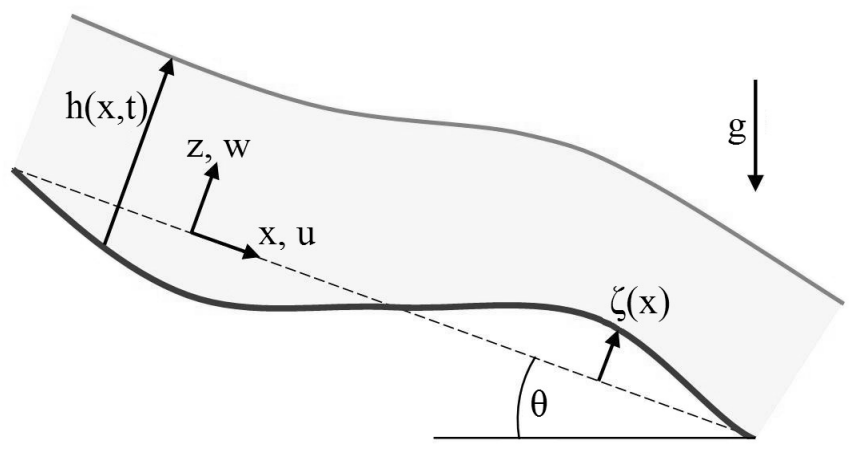

Figure 1: The coordinate system and flow setup. 
Navier-Stokes equations using the computational fluid dynamics package CFX. In this comparison, bottom topography is included. The combined effect of surface tension and bottom topography on the stability is briefly discussed. Finally, the weighted residual model, which turns out to be the optimal model, is then compared to experimental results for flow over a wavy incline.

\section{Description of models}

Three models are considered in this study. They are the shallow-water model, the integral-boundary-layer model, and the weighted residual model. All three of the models are given below, with a brief description of how they are obtained.

As suggested by the name, the shallow-water model is founded on shallow-water theory and hence assumes that the fluid is incompressible and inviscid, and that the thickness of the fluid is much smaller than the characteristic length in the direction of the flow. It then follows that the pressure distribution is hydrostatic and that the streamwise velocity is depth independent. This model is limited to gentle inclines.

After these simplifications are made, three modifications are added to make the model more realistic. A flow factor multiplying the advective term is added; the value is empirically determined, and depends on whether the flow is laminar or turbulent. For the laminar model, a value of $4 / 5$ is used [1]. A term partially accounting for viscosity and a bottom friction term are also added.

Two different forms of the shallow-water equations have been developed: one pertaining to laminar flow and the other to turbulent flow. The difference between the two is in the viscosity parameter of the added viscous term, the form of the bottom friction term, and the coefficient of the advection term. Balmforth and Mandre [1] give a thorough description of the two versions of the model. The laminar model is used in this study because flows having a Reynolds number of order unity are considered, and because the other two models are developed assuming laminar flow. The equations describing the laminar shallow-water model are given by

$$
\begin{gathered}
\frac{\partial h}{\partial t}+\frac{\partial q}{\partial x}=0 \\
\frac{\partial q}{\partial t}+\frac{\partial}{\partial x}\left(\frac{4}{5} \frac{q^{2}}{h}+\frac{\cot \theta}{2 R e} h^{2}\right)=-\frac{1}{5} \frac{q}{h} \frac{\partial q}{\partial x}-\frac{\cot \theta}{R e} h \frac{d \zeta}{d x} \\
+\frac{1}{\delta R e}\left(h-\frac{q}{h^{2}}\right)+\delta^{2} h W e \frac{\partial^{3}}{\partial x^{3}}(\zeta+h) \\
+\frac{\delta}{R e}\left(\frac{\partial^{2} q}{\partial x^{2}}-\frac{q}{h} \frac{\partial^{2} h}{\partial x^{2}}-\frac{1}{h} \frac{\partial h}{\partial x} \frac{\partial q}{\partial x}+\frac{q}{h^{2}}\left(\frac{\partial h}{\partial x}\right)^{2}\right)
\end{gathered}
$$

The non-dimensional flow variables are $h$, the height of the free surface from the bottom, and $q$, the mass flow rate. Here, $\theta$ denotes the angle of inclination of the surface, $R e$ is the Reynolds number, defined as $R e=Q / \nu$, and $W e$ is the Weber number, defined as $W e=\frac{\sigma H}{\rho Q^{2}}$, where $Q$ is the non-dimensional characteristic 
volume flow rate, $H$ is the Nusselt thickness, $\rho$ is the fluid density, $\sigma$ is the surface tension coefficient, and $\nu$ is the kinematic viscosity of the fluid. The shallowness parameter, $\delta$, is the ratio of the Nusselt thickness of the fluid to some characteristic length in the $x$ direction, and is assumed to be small.

The integral-boundary-layer model is derived more rigorously from the NavierStokes equations. The non-dimensionalized continuity and momentum equations are

$$
\begin{gathered}
\frac{\partial u}{\partial x}+\frac{\partial w}{\partial z}=0 \\
\delta R e\left(\frac{\partial u}{\partial t}+u \frac{\partial u}{\partial x}+w \frac{\partial u}{\partial z}\right)=-\delta R e \frac{\partial p}{\partial x}+3+\delta^{2} \frac{\partial^{2} u}{\partial x^{2}}+\frac{\partial^{2} u}{\partial z^{2}}, \\
0=-\operatorname{Re} \frac{\partial p}{\partial z}-3 \cot \theta+\delta \frac{\partial^{2} w}{\partial z^{2}} .
\end{gathered}
$$

Here, the advective terms in the z-momentum equation are neglected because they become third order in $\delta$ when the pressure is substituted into the $\mathrm{x}$-momentum equation. The model is therefore second-order accurate in $\delta$. This model more accurately accounts for the fluid viscosity and allows a non-hydrostatic pressure distribution, which are improvements over the shallow-water model.

At the free surface, we apply dynamic and kinematic conditions, given in nondimensional form by

$$
\left.\begin{array}{c}
0=p-\frac{2 \delta}{R e} \frac{\partial w}{\partial z}+\delta^{2} W e \frac{\partial^{2}}{\partial x^{2}}(h+\zeta) \\
0=\frac{\partial u}{\partial z}-4 \delta^{2} \frac{\partial}{\partial x}(h+\zeta) \frac{\partial u}{\partial x}+\delta^{2} \frac{\partial w}{\partial x}
\end{array}\right\} \begin{gathered}
\text { at } z=h+\zeta \\
\left.w=\frac{\partial h}{\partial t}+u \frac{\partial(h+\zeta)}{\partial x}\right\} \text { at } z=h+\zeta .
\end{gathered}
$$

As well, the following no-slip conditions are imposed at the bottom surface:

$$
u=w=0 \text { at } z=\zeta .
$$

The pressure can be eliminated by integrating the z-momentum equation and using the first condition in eqn. (6) to find an expression for pressure, and then substituting this expression into the $\mathrm{x}$-momentum equation. This leaves the continuity equation and a single momentum equation. The form of the streamwise velocity is then assumed based on the known steady flow over an even-bottom inclined plane, and modified to account for bottom topography defined by $\zeta(x)$. The profile is given by 


$$
u=\frac{3 q}{2 h^{3}}\left(2(h+\zeta) z-z^{2}-\zeta^{2}-2 h \zeta\right) .
$$

The $\mathrm{z}$ dependence is then eliminated by integrating the equations across the fluid layer and applying the boundary conditions. The final form of the integralboundary-layer model equations are

$$
\begin{gathered}
\frac{\partial h}{\partial t}+\frac{\partial q}{\partial x}=0 \\
\frac{\partial q}{\partial t}+\frac{\partial}{\partial x}\left(\frac{6}{5} \frac{q^{2}}{h}+\frac{3}{2} \frac{\cot \theta}{R e} h^{2}\right)=\delta^{2} h W e \frac{\partial^{3}}{\partial x^{3}}(h+\zeta) \\
-3 h \frac{\cot \theta}{R e} \frac{\partial \zeta}{\partial x}+\frac{3}{\delta R e}\left(h-\frac{q}{h^{2}}\right) \\
+\frac{\delta}{R e}\left(\frac{9}{2} \frac{\partial^{2} q}{\partial x^{2}}-\frac{6}{h} \frac{\partial h}{\partial x} \frac{\partial q}{\partial x}-\frac{3}{h} \frac{\partial q}{\partial x} \frac{d \zeta}{d x}+3 \frac{q}{h^{2}} \frac{\partial h}{\partial x} \frac{d \zeta}{d x}\right) \\
+\frac{\delta}{R e}\left(6 \frac{q}{h^{2}}\left(\frac{\partial h}{\partial x}\right)^{2}-6 \frac{q}{h^{2}}\left(\frac{d \zeta}{d x}\right)^{2}-6 \frac{q}{h} \frac{\partial^{2} h}{\partial x^{2}}-\frac{9}{2} \frac{q}{h} \frac{d^{2} \zeta}{d x^{2}}\right) .
\end{gathered}
$$

The weighted residual model is derived following a similar procedure to that used for the integral-boundary layer model. However, before integrating in the cross-stream direction, the equations are multiplied by a weighting function; in this case, a parabolic profile is used as the weighting function. In this way, a weighted average over the depth of the fluid is used. The resulting model equations are

$$
\begin{gathered}
\frac{\partial h}{\partial t}+\frac{\partial q}{\partial x}=0 \\
\frac{\partial q}{\partial t}+\frac{\partial}{\partial x}\left(\frac{9}{7} \frac{q^{2}}{h}+\frac{5}{4} \frac{\cot \theta}{R e} h^{2}\right)=\frac{5}{6} \delta^{2} h W e \frac{\partial^{3}}{\partial x^{3}}(h+\zeta) \\
+\frac{q}{7 h} \frac{\partial q}{\partial x}-\frac{5 h}{2} \frac{\cot \theta}{R e} \frac{d \zeta}{d x}+\frac{5}{2 \delta R e}\left(h-\frac{q}{h^{2}}\right) \\
+\frac{\delta}{R e}\left(\frac{9}{2} \frac{\partial^{2} q}{\partial x^{2}}-\frac{9}{2 h} \frac{\partial h}{\partial x} \frac{\partial q}{\partial x}-\frac{5}{2} \frac{q}{h^{2}} \frac{\partial h}{\partial x} \frac{d \zeta}{d x}+4 \frac{q}{h^{2}}\left(\frac{\partial h}{\partial x}\right)^{2}\right) \\
+\frac{\delta}{R e}\left(-5 \frac{q}{h^{2}}\left(\frac{d \zeta}{d x}\right)^{2}-6 \frac{q}{h} \frac{\partial^{2} h}{\partial x^{2}}-\frac{15}{4} \frac{q}{h} \frac{d^{2} \zeta}{d x^{2}}\right)
\end{gathered}
$$

\section{Model performance}

To evaluate and compare the performance of the three models, four methods are employed. First, the critical Reynolds number at which the flow becomes unstable 
is calculated for the flat bottom case, and compared to the known theoretical value. Second, the neutral stability curves for each model are compared to experimental data collected by Liu et al. [4]. Next, the evolution of the flow rate q, as predicted by the models, is compared to the solution of the full Navier-Stokes equations, where the solution to the full Navier-Stokes equations was obtained using the software package CFX. Finally, critical Reynolds number predictions for the weighted residual model for flow over a wavy bottom are compared to experimental results collected by Wierschem et al. [5]. Only the weighted residual model is considered in this final comparison because it is found to perform the best in the previous three tests.

The critical Reynolds number for a thin film flow down an even-bottom inclined plane has been determined by Benjamin [6] and Yih [7] from the corresponding Orr-Sommerfeld equation. The result is that the critical Reynolds number is:

$$
R e_{\text {crit }}=\frac{5}{6} \cot \theta \text {. }
$$

Performing a linear stability analysis on the shallow-water model, the integralboundary-layer model, and the weighted residual model yields the following results:

$$
\begin{aligned}
R e_{\text {crit }}^{S W M} & =\frac{5}{22} \cot \theta, \\
R e_{\text {crit }}^{I B L} & =\cot \theta,
\end{aligned}
$$

and

$$
R e_{c r i t}^{W R M}=\frac{5}{6} \cot \theta
$$

respectively.

These results show that, of the three models considered, only the weighted residual model correctly predicts the critical Reynolds number. The integral-boundarylayer model predicts a critical Reynolds number slightly higher than the correct value, although it is still close. The shallow-water model gives a very poor prediction, significantly underestimating the critical Reynolds number.

To further evaluate the performance of the three models, the neutral stability curves for each of the models are compared to experimental data gathered by Liu et al. [4] and plotted in Figure 2.

As with the critical Reynolds number predictions, the weighted residual model most closely matches the experimental data. The integral-boundary-layer model is a slightly poorer predictor of the experimental data, although it gives a much better prediction than the shallow-water model. It should also be noted that the experimental data is for a very gentle incline, which is much more appropriate for the shallow-water model than for the integral-boundary-layer or weighted residual models; despite this, the weighted residual model does very well.

For the next comparison, numerical solutions using the models are compared to direct numerical simulations of the full Navier-Stokes equations. The full NavierStokes equations for the free surface flow down an inclined plane have been solved 


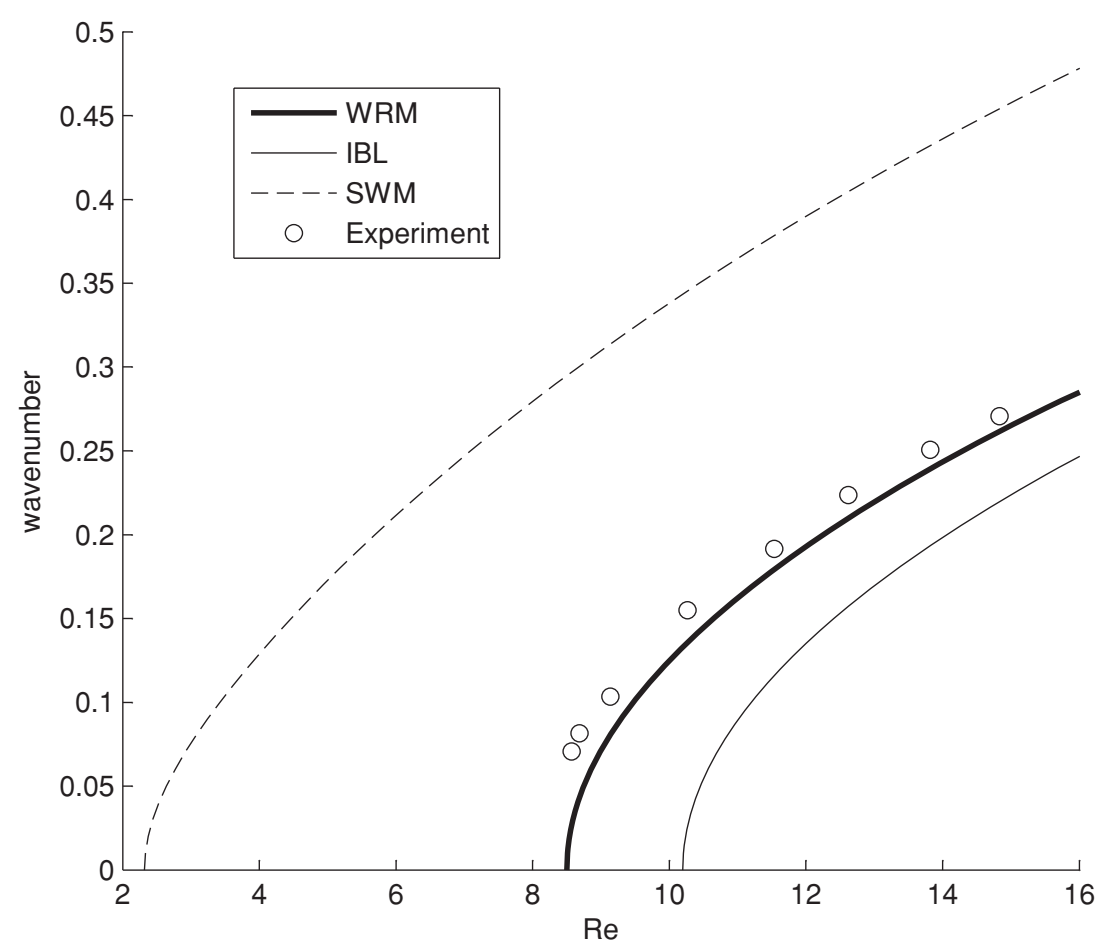

Figure 2: Comparison of neutral stability curves with experimental data for a glycerin-water solution with $\theta=5.6^{\circ}$.

by employing CFX. This software package solves the full Navier-Stokes equations using a combination of finite volume and finite element methods [8]. The location of the interface is determined using a compressive volume fraction advection scheme [9].

The models considered in this study can also be used to predict the transient behaviour of the flow by numerically solving the equations. Using LeVeque's fractional step method [10], the unsteady equations for each model can be solved. When the steady-state solution, found using the bvp4c routine in matlab, is used as the initial condition and is perturbed, waves develop on the free surface. Eventually, characteristic features such as the number and height of the wave peaks in a domain of a given length are maintained. The wave characteristics predicted by the weighted residual and integral-boundary-layer models are compared to those found by solving the full Navier-Stokes equations. The shallow-water model is not included in this comparison because the inclination considered is too large for that model.

The comparison between the solutions to the full Navier-Stokes equations and the model equations is for a case without surface tension (i.e. $W e=0$ ). The angle of inclination is $33.7^{\circ}$, and the Reynolds number is 2.28 . The number and 

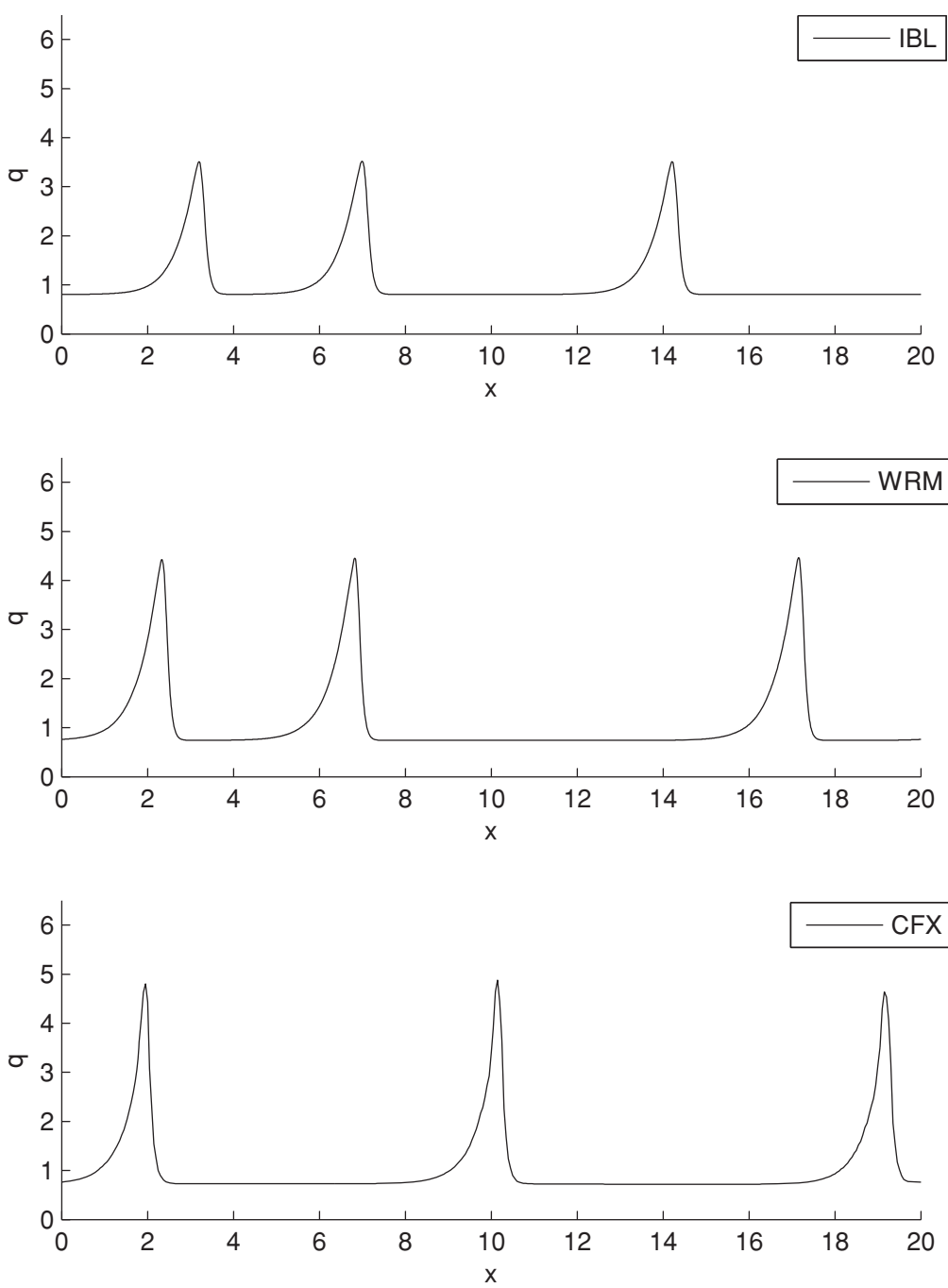

Figure 3: Fully developed roll waves for the IBL model, the WRM, and CFX; volume flow rate versus position along domain.

height of roll waves emerging from the weighted residual and integral-boundarylayer models are shown in Figure 3, where the non-dimensional volume flow rate is plotted against the position along the domain. The calculations for the same flow, resulting from the full Navier-Stokes equations using CFX, are also shown in Figure 3. Figure 4 shows the fluid height and bottom topography for both models and the CFX results, for the same set of parameters.

Comparing the figures, it can be seen that both the integral-boundary-layer and the weighted residual models correctly predict the number of roll waves in the 

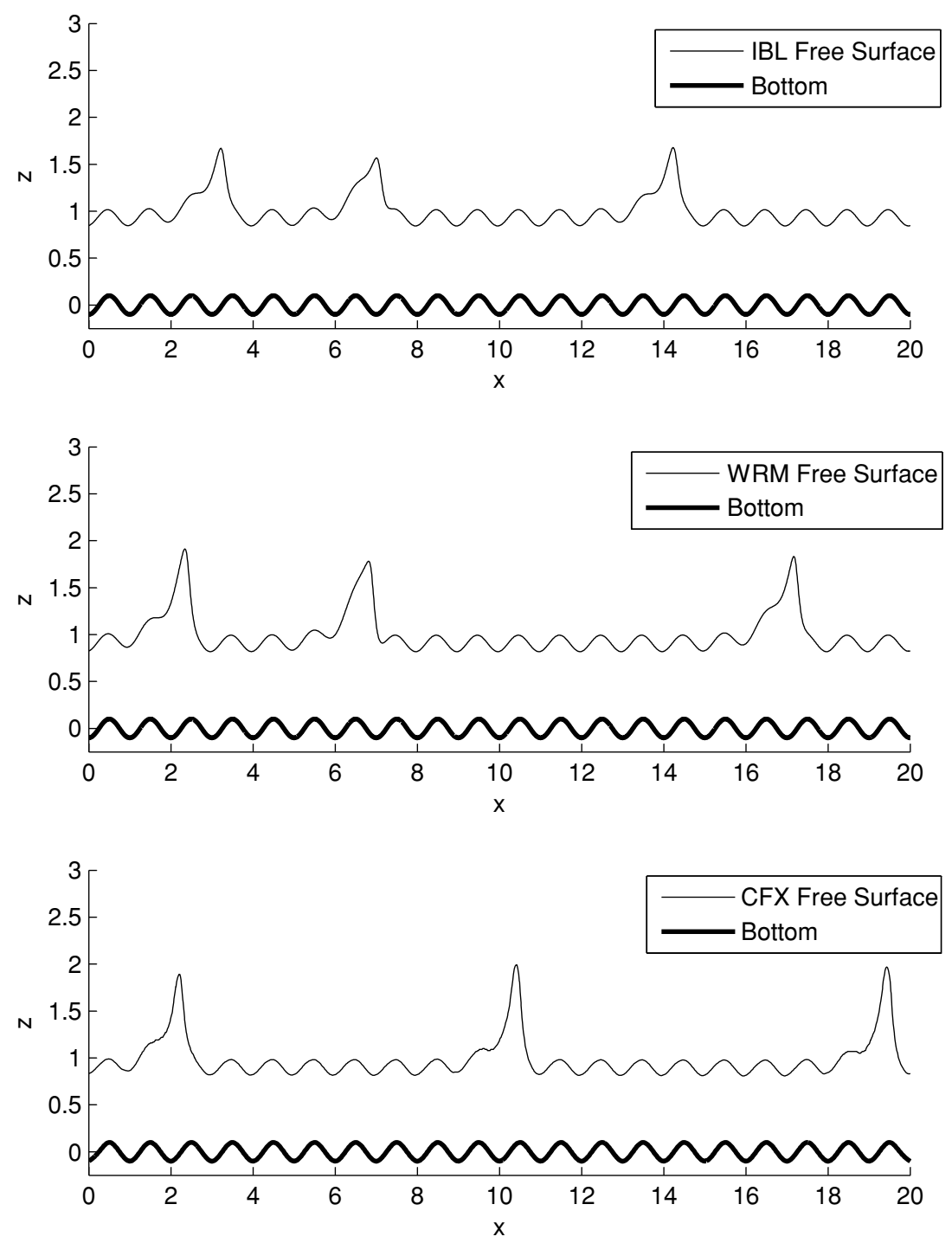

Figure 4: Fully developed roll waves for the IBL model, the WRM, and CFX; fluid height versus position along domain.

Comparing the figures, it can be seen that both the integral-boundary-layer and the weighted residual models correctly predict the number of roll waves in the domain. However, the weighted residual model more closely predicts the height of the waves.

For the case shown in Figure 3, tall sharp peaks emerge. If surface tension would have been included, the waves that would develop would be smoother and 
Table 1: Comparison between experimental, numerical and theoretical values of $R e_{c r i t}$ for a wavy-incline case with $\delta=0.1$.

\begin{tabular}{|l|l|c|c|c|}
\hline \multirow{2}{*}{$\theta$} & \multirow{2}{*}{$R_{\text {crit }}^{\text {even }}$} & \multicolumn{3}{|c|}{ Ex $_{\text {crit }}$} \\
\cline { 3 - 5 } & & Experimental & Numerical & Theoretical \\
\hline $15^{\circ}$ & 3.3 & $5.1 \pm 0.4$ & $(5.5,5.6)$ & 5.6 \\
\hline $30^{\circ}$ & 1.4 & $2.2 \pm 0.2$ & $(1.8,1.9)$ & 1.7 \\
\hline $40.7^{\circ}$ & 0.97 & $1.3 \pm 0.1$ & $(1.1,1.2)$ & 1.1 \\
\hline
\end{tabular}

wider. Surface tension and bottom topography also affect the stability of the flow by altering the critical Reynolds number. D'Alessio et al. [2] have shown that for small to moderate surface tension, bottom topography increases the critical Reynolds number of the flow while for larger surface tension and topography, the opposite occurs.

As a final confirmation of the validity of the weighted residual model, critical Reynolds number predictions are compared to experimental data for a wavy incline, collected by Wierschem et al. [5]. Table 1 contrasts the experimentally obtained critical Reynolds numbers with those from the weighted residual model for three different angles of inclination. Presented in the table are results from the numerical simulations along with theoretical values obtained from a linear stability analysis of the weighted residual model. Also listed in the table is $R e_{c r i t}^{\text {even }}$, the corresponding critical Reynolds number for an even bottom. The table shows that numerical predictions are almost all within the error of the experiment, and in close agreement with the theoretical values. This further demonstrates that the weighted residual model is the optimal model for flow over an incline with or without bottom topography.

\section{Summary}

This study compared the performance of three models for free surface flow down a wavy inclined plane. The simplest model is the shallow-water model, and it gives the poorest agreement with theory and experiments. The integral-boundarylayer model provides much better predictions in both areas, although the critical Reynolds number still does not match the theoretical value for an even bottom. The weighted residual model exactly predicts the theoretical critical Reynolds number, and also matches the experimental neutral stability curve very closely.

The integral-boundary-layer and weighted residual models were solved numerically and compared to direct numerical simulations of the full NavierStokes solutions, obtained using CFX. The weighted residual model performs slightly better than the integral-boundary-layer model since it yields better agreement in the height of the peaks. 
Finally, the weighted residual model gives good predictions for the critical Reynolds number for flow over a wavy surface when compared to experimental data. Considering all four methods of comparison, the weighted residual model gives the best results over all.

\section{Acknowledgements}

Financial support for this research was provided by the Natural Sciences and Engineering Research Council of Canada.

\section{References}

[1] Balmforth, N. J. \& Mandre, S., Dynamics of roll waves, J. Fluid Mech. 514, pp. 1-33, 2004.

[2] D’Alessio, S.J.D., Pascal, J.P. \& Jasmine, H., Instability in gravity-driven flow over uneven surfaces, Physics of Fluids 21, pp. 062105-1 - 062105-11, 2009.

[3] Ruyer-Quil, C. \& Manneville, P., Further accuracy and convergence results on the modeling of flows down inclined planes by weighted residual approximations, Physics of Fluids 14, pp. 170-183, 2002.

[4] Liu, J., Paul, J.D. \& Gollub, J.P., Measurements of the primary instabilities of film flows, J. Fluid Mech. 250, pp. 69-101, 1993.

[5] Wierschem, A., Lepski, C., Aksel, N., Effect of long undulated bottoms on thin gravity-driven films, Acta Mechanica 179, pp. 41-66, 2005.

[6] Benjamin, T.B., Wave formation in laminar flow down an inclined plane, $J$. Fluid Mech. 2, pp. 554-574, 1957.

[7] Yih, C-S., Stability of liquid flow down an inclined plane, The Physics of Fluids 6, pp. 321-334, 1963.

[8] ANSYS, Inc., ANSYS CFX Solver Theory Guide, ANSYS CFX Release 11.0, ANSYS Europe, Ltd, 2006, pp. 277-295.

[9] Zwart, P.J., Numerical modelling of free surface and cavitating flows, VKI Lecture Series, ANSYS Canada Ltd, 2005.

[10] LeVeque, R. J., Finite volume methods for hyperbolic problems, Cambridge University Press, Cambridge, UK, 2002. 